\title{
Cancers with Higher Density of Tumor-Associated Macrophages Were Associated with Poor Survival Rates
}

\author{
Kyong Yeun Jung ${ }^{1,2} \cdot$ Sun Wook Cho ${ }^{1,3}$ \\ Young A Kim ${ }^{4}$. Daein $\mathrm{Kim}^{3}$ \\ Byung-Chul Oh ${ }^{5}$. Do Joon Park ${ }^{1}$ \\ Young Joo Park ${ }^{1}$ \\ ${ }^{1}$ Department of Internal Medicine, Seoul National \\ University College of Medicine, Seoul; \\ 2Department of Internal Medicine, \\ Eulji University School of Medicine, Seoul; \\ ${ }^{3}$ Department of Internal Medicine, \\ National Medical Center, Seoul; ${ }^{4}$ Department of \\ Pathology, SMG-SNU Boramae Medical Center, \\ Seoul; ${ }^{5}$ Lee Gil Ya Cancer and Diabetes Institute, \\ Gachon University Graduate School of Medicine, \\ Incheon, Korea
}

\author{
Received: May 21, 2015 \\ Accepted: June 12015 \\ Corresponding Author \\ Young Joo Park, MD, PhD \\ Department of Internal Medicine, Seoul National \\ University College of Medicine, 101 Daehak-ro, \\ Jongno-gu, Seoul 110-799, Korea \\ Tel: $+82-2-2072-4183$ \\ Fax: +82-2-764-2199 \\ E-mail: yjparkmd@snu.ac.kr
}

Background: Macrophages are a component of a tumor's microenvironment and have various roles in tumor progression and metastasis. This study evaluated the relationships between tumorassociated macrophage (TAM) density and clinical outcomes in 14 different types of human cancers. Methods: We investigated TAM density in human tissue microarray sections from 14 different types of human cancers $(n=266)$ and normal thyroid, lung, and breast tissues $(n=22)$. The five-year survival rates of each cancer were obtained from the 2011 Korea Central Cancer Registry. Results: Among 13 human cancers, excluding thyroid cancer, pancreas, lung, and gallbladder cancers had the highest density of CD163-positive macrophages $(7.0 \pm 3.5 \%, 6.9 \pm 7.4 \%$, and 6.9 $\pm 5.5 \%$, respectively). The five-year relative survival rates of these cancers (pancreas, $8.7 \%$; lung, $20.7 \%$; gallbladder, $27.5 \%$ ) were lower than those of other cancers. The histological subtypes in thyroid cancer exhibited significantly different CD163-positive macrophages densities (papillary, $1.8 \pm 1.6 \%$ vs anaplastic, $22.9 \pm 17.1 \% ; p<.001$ ), but no significant difference between histological subtypes was detected in lung and breast cancers. Moreover, there was no significant difference in CD163-positive macrophages densities among the TNM stages in lung, breast, and thyroid cancers. Conclusions: Cancers with higher TAM densities (pancreas, lung, anaplastic thyroid, and gallbladder) were associated with poor survival rate.

Key Words: Tumor-associated macrophage; Prognosis; Neoplasms
The tumor microenvironment includes cancer cells and various stromal cells, including immune cells, fibroblasts, and vascular endothelial cells. ${ }^{1}$ Tumor-associated macrophages (TAMs) are also present in the tumor microenvironment and are important in tumor progression and metastasis. ${ }^{2}$ TAMs can induce neoplastic cell transformation, elicit tumor destructive reactions, and have either negative or positive effects on tumor growth. ${ }^{3}$ Several reports have suggested that TAMs are associated with tumor growth, disease progression, and poor prognosis in some human cancers. ${ }^{4-6}$ Moreover, high densities of TAMs are present in the more advanced stages of cancers that have poor prognoses, such as breast, ${ }^{7}$ lung, ${ }^{8}$ thyroid, ${ }^{5}$ and bladder ${ }^{3}$ cancers. In contrast, several reports, including those on colorectal, ${ }^{9}$ stomach, ${ }^{10}$ lung, ${ }^{11,12}$ and endometrial ${ }^{13}$ cancers, have shown that a high density of TAMs is associated with a high survival rate. Collectively, these results suggest that TAMs can have either positive or negative effects depending on the specific tissue type, tumor location, and tumor stage. The aim of this study was to evaluate the relationship between TAM density and clinical outcome in various human cancers.

\section{MATERIALS AND METHODS}

\section{Study subjects and tissue microarrays}

We purchased 14 different type of human cancer human tissue microarray sections (lung, 49; breast, 49; thyroid, 10; pancreas, 10; gallbladder, 9; larynx, 9; esophagus, 10; liver, 10; cervix, 10; ovary, 10; stomach, 10; prostate, 9; kidney, 9; and endometrium, 9 sections) and normal tissues (lung, 9; breast, 8 sections) from SuperBioChips Laboratories (Seoul, Korea). The supplier pro- 
vided the clinical information associated with each section, including age at surgery; gender; pathologic diagnosis; TNM staging for cancers; survival/death follow-up result for lung and breast cancers; and estrogen receptor (ER), progesterone receptor (PR), p53, and C-erbB2 expression for breast cancers. Thyroid microarrays contained normal thyroid $(n=5)$, papillary thyroid cancer (PTC; $n=35$ ), and anaplastic thyroid cancer (ATC; $n=18$ ), and one of the thyroid microarray slides was used in a previous study. ${ }^{14}$ Clinical and pathologic data for the thyroid samples were obtained from medical records at Seoul National University Hospital and SMG-SNU Boramae Medical Center. The five-year cancer relative survival rates were obtained from the 2011 annual report of cancer statistics in Korea. ${ }^{15}$

\section{Immunohistochemistry on tissue array blocks}

CD68 and CD163 were used as TAM markers. Immunohistochemical (IHC) staining for CD68 and CD163 was performed using the BenchMark XT Slide Preparation System (Ventana Medical Systems, Tucson, AZ, USA) and CD68 (ready-to-use, 514H12, Novocastra, Newcastle upon Tyne, UK) and CD163 (1:200, 10D6, ER2, Novocastra). The proportion of CD163positive area in each tumor was evaluated after IHC staining (Fig. 1). We divided the area of each tissue core into quarters and a central area and randomly chose all five fragments to determine the positive stain proportion (Fig. 1A). Areas of fibrosis or tumor necrosis among the randomly chosen fragments were excluded. We used a color deconvolution plug-in for Image J software to identify the positive stains and to calculate the percent CD163-positive area (Fig. 1B, C). TAM density was determined by calculating the average CD163-positive area (\%) at a minimum of four different sites in each tissue.

\section{Statistical analysis}

The CD163-positive macrophages density $(\%)$ is presented as mean \pm standard deviation. Differences in CD163-positive macrophages densities among TNM cancer stages, histological subtypes, and pathological characteristics were determined using one-way analysis of variance (ANOVA) or Student's t test. Statistical significance is indicated by a p-value less than .05 . All data were analyzed with IBM SPSS Statistics ver. 20.0 (SPSS Inc., Chicago, IL, USA).

\section{Ethics statement}

This study was approved by the Institutional Review Boards of Seoul National University Hospital (1107-060-369) and SMGSNU Boramae Medical Center (06-2010-176). The need for informed consent was waived by those boards.

\section{RESULTS}

\section{CD163-positive macrophage densities and prognoses in human cancers}

The average CD163-positive area was significantly correlated with the average CD68-positive area $(\%)$ in thyroid $(r=0.775, p<$ $.001)$ (Appendix 1A), breast $(\mathrm{r}=0.806, \mathrm{p}<.001)$ (Appendix 1B), and lung $(r=0.780, p<.001)$ (Appendix 1C) cancers. Therefore, our remaining IHC assessments were performed with CD163 data.

The CD163-positive macrophages densities and the five-year relative survival rates in the 14 different types of human cancers are summarized in Fig. 2. Excluding thyroid cancer, of the 13 other human cancers, pancreas, lung, and gallbladder cancers had the highest density of CD163-positive macrophages (7.0 \pm
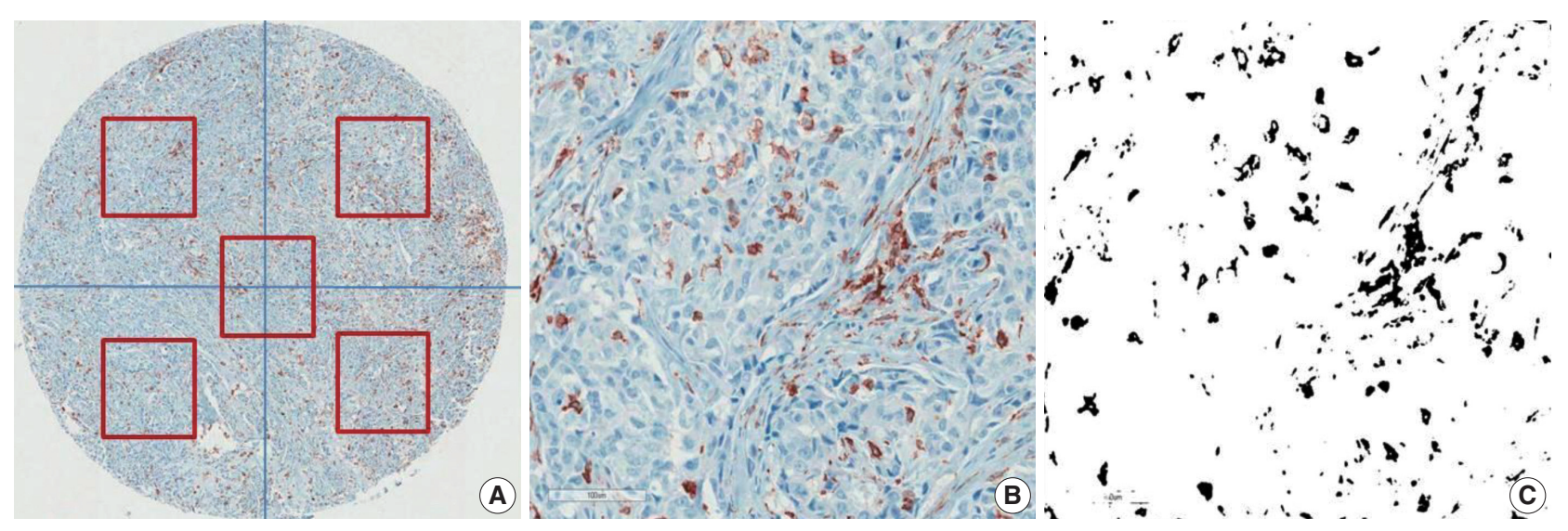

Fig. 1. Immunohistochemical staining for CD163 in breast cancer. Representative CD163 staining in breast cancer. TAM density was measured by averaging the CD163-positive area (\%) of five different sites in each tissue (A). The positive IHC staining area (\%) was separated and calculated using the Image $\mathrm{J}$ color deconvolution plugin $(\mathrm{B}, \mathrm{C})$. 


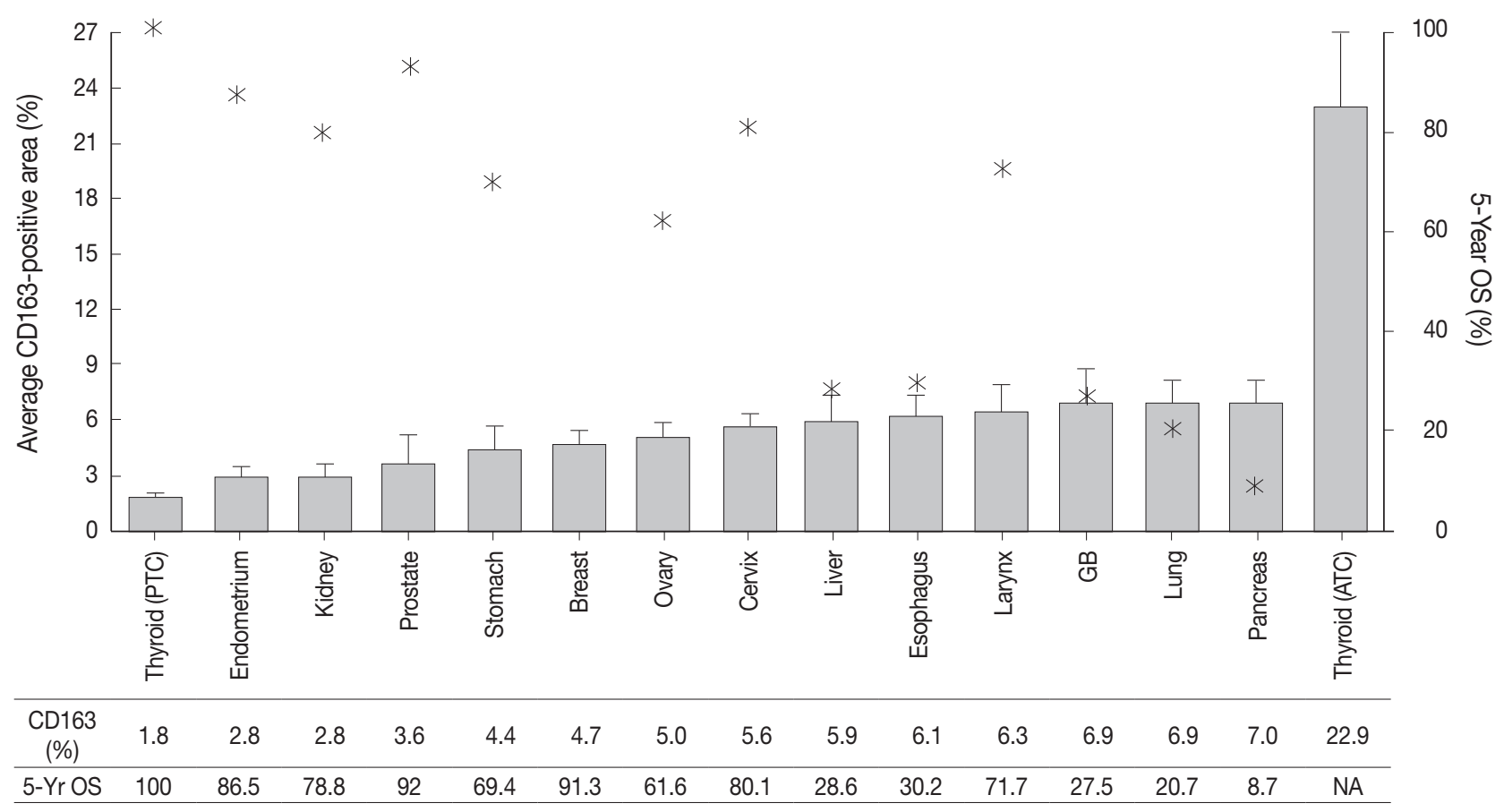

Fig. 2. CD163-positive macrophage densities and five-year survival rates in 14 different types of human cancers. Left axis and bar graphs represent the average CD163-positive area (\%) as mean \pm standard error. Right axis and star-shaped markers represent the five-year overall survival (OS, \%) obtained from the 2011 Annual Report of Cancer Statistics in Korea. PTC, papillary thyroid cancer; GB, gallbladder; ATC, anaplastic thyroid cancer; NA, not acquired.

$3.5 \%, 6.9 \pm 7.4 \%$, and $6.9 \pm 5.5 \%$, respectively). In contrast, endometrium, prostate, and kidney cancers had the lowest densities of CD163-positive macrophages $(3.6 \pm 4.6 \%, 2.8 \pm 2.5 \%$, and $2.8 \pm 1.8 \%$, respectively). Interestingly, among the tested cancers, five-year relative survival rate (\%) was inversely correlated with CD163-positive macrophages density. The five-year overall survival rates of the cancers with highest TAM densities (pancreas, 8.7\%; lung, $20.7 \%$; gallbladder and biliary tract, $27.5 \%$ ) were lower than those of the cancers with the lowest TAM densities (endometrial, 86.5\%; kidney, 78.8\%; prostate, 92\%).

Of the 14 cancers assessed, thyroid cancer exhibited an extremely wide range of TAM densities that varied according to pathologic subtype (Fig. 3). The ATC cases had the highest density of CD163-positive macrophages $(22.9 \pm 17.1 \%)$, whereas the PTC cases had the lowest CD163-positive macrophage density $(1.8 \pm 1.3 \%)$.

Clinicopathological correlations with TAM density in thyroid, lung, and breast cancers

To investigate the role of TAMs in human cancers, we evaluated the correlations between TAM density and clinicopathological features in lung, breast, and PTC cancers. The CD163-positive macrophage density was significantly higher in cancer tissues

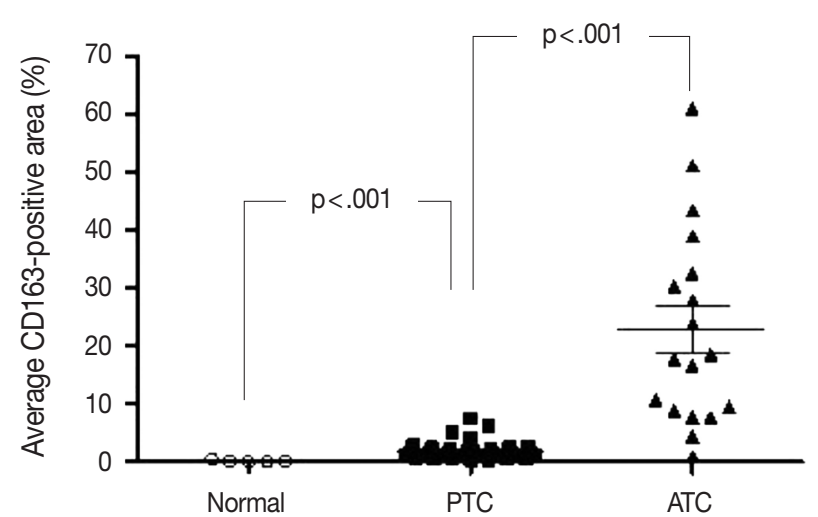

Fig. 3. The CD163-positive macrophage densities of normal tissue, papillary thyroid cancer (PTC) and anaplastic thyroid cancer (ATC).

than normal tissues in breast $(4.7 \pm 4.5 \%$ vs $0.6 \pm 0.2 \%$; $\mathrm{p}<.001)$, lung $(6.9 \pm 7.4 \%$ vs $1.6 \pm 1.6 \% ; \mathrm{p}<.001)$, and thyroid (PTC, $1.8 \pm$ $1.3 \%$ vs normal, $0.2 \pm 0.2 \%$; $<<.001$ ) tissues (Fig. 3). Among the histological subtypes of lung (adenocarcinoma, $5.8 \pm 4.4 \%$ vs squamous cell carcinoma, $5.9 \pm 4.5 \%$ vs large cell carcinoma, $8.7 \pm$ $7.3 \% ; \mathrm{p}=.428$ ) and breast cancers (infiltrating ductal carcinoma, $4.0 \pm 3.4 \%$ vs medullary carcinoma, $9.3 \pm 3.4 \% ; \mathrm{p}=.055)$, there were no significant differences in CD163-positive macrophages density (Appendix 2). Differences among TNM stages were ev- 
aluated by assessing adenocarcinoma and squamous cell carcinoma of lung cancer, infiltrating ductal carcinoma of breast cancer, and PTC. There were no significant differences in CD163-positive macrophage density associated with TNM stage in lung $(\mathrm{p}=$ $.821)$, breast $(\mathrm{p}=.060)$, or thyroid (PTC, $\mathrm{p}=.943)$ cancer (Appendix 2). Within the breast cancer samples, several prognostic markers including ER, PR, p53, and C-erbB2 were analyzed. The ER-positive $(3.4 \pm 4.5 \%$ vs $5.6 \pm 4.8 \% ; \mathrm{p}=.182)$ and PRpositive $(3.7 \pm 4.4 \%$ vs $5.4 \pm 4.9 \% ; \mathrm{p}=.319)$ breast cancers showed non-significant trends toward lower CD163-positive macrophages density than those in the ER- or PR-negative breast cancers. In contrast, p53-positive $(5.9 \pm 5.9 \%$ vs $4.2 \pm 3.6 \%$; $=$ .269) and C-erbB2-positive $(6.4 \pm 6.8 \%$ vs $4.4 \pm 3.8 \%$; $\mathrm{p}=.233)$ breast cancer showed nonsignificant trends toward higher CD163positive macrophage density than those in $\mathrm{p} 53$ - or C-erbB2-negative breast cancers.

\section{DISCUSSION}

Our results demonstrate that CD163-positive macrophage density is inversely correlated with five-year cancer survival rate in a variety of human cancers. This finding suggests the presence of a potential pro-tumorigenic role of TAMs in some human cancers. Among the 14 different types of human cancers assessed, ATC, the most aggressive cancer in humans, ${ }^{16,17}$ had the highest CD163-positive macrophages density (23\%), which was markedly higher than the second highest TAM density (7\%) detected in the pancreas cancer samples. ATC accounts for up to $2 \%$ of thyroid cancers and is characterized by an extremely poor survival rate with a one-year average survival rate of $20 \%$ and a median survival duration of five months. ${ }^{18,19}$ In contrast, the most common well-differentiated thyroid cancer, PTC, had the lowest CD163-positive macrophages density $(1.8 \%)$. PTC is associated with lower recurrence and mortality rates ${ }^{20}$ than other solid and relatively indolent tumors and has a five-year survival rate higher than $90 \% .{ }^{15}$ Since the differentiation of pathologic subtypes is distinctive and survival rates markedly differ among subtypes, our observation of a striking difference in CD163-positive macrophages density between two thyroid cancer subtypes led us to hypothesize that TAM density is a potential prognostic factor in human cancers.

In contrast, histological subtypes of lung and breast cancers did not exhibit significant differences in CD163-positive macrophage density. Correlations between TAM density and clinicopathological features have been reported in ductal and lobular carcinomas in breast cancer ${ }^{21,22}$ and in adenocarcinoma ${ }^{23,24}$ and non-small cell $^{25,26}$ lung cancers. Nonetheless, only a few reports have compared TAM density based on histological subtype. Although our data did not detect a significant difference related to histological subtype in lung or breast cancers, the number of each subtype in our sample population was limited. Therefore, we recommend a larger-scale study of TAM density in a variety of histological subtypes.

In this study, there were no significant differences in CD163positive macrophage density among TNM stages in lung, breast, or thyroid cancers. In addition, cancer tissues showed higher CD163-positive macrophage densities than those in normal tissues in lung, breast, and thyroid samples. In breast cancer, there were trends toward negative correlations of TAM density with ER and PR expression, whereas there were trends toward positive correlations of TAM density with p53 and C-erbB2 expression. Previous reports indicate that TAM infiltration is inversely correlated with ER expression in breast cancer. ${ }^{7,27}$ However, this correlation might vary with TAM location. ${ }^{22}$ Medrek et al. ${ }^{22}$ reported that a dense infiltration of CD163-positive macrophages in tumor stroma was associated with ER- and PR-negativity, but there was no such association in tumor nest. Our study results show a non-significant trend toward negative correlations between ER and PR expressions and density of CD163-positive macrophages. Due to data limitations, we could not assess such correlations according to infiltration location.

Selecting the most appropriate marker for TAM assessment can be challenging. Originally, a TAM was seen as a residual macrophage within the tumor microenvironment, and the biological characteristics of TAMs have been shown to be anti-tumorigenic, cytotoxic, or pro-tumorigenic. ${ }^{1,28}$ Furthermore, macrophages can undergo polarization and change from an M1-macrophage (classically activated, pro-inflammatory) to an M2macrophage (alternatively activated, anti-inflammatory or regenerative). ${ }^{29}$ Collectively, these findings indicate the difficulty in choosing a cancer-specific TAM marker. In the beginning of this study, we performed IHC investigations using both CD68, a pan macrophage marker, ${ }^{29}$ and CD163, a more specific marker for M2-macrophages. ${ }^{30}$ Those investigations revealed that the CD163-positive areas were significantly positively correlated with the CD68-positive areas in lung, breast, and thyroid cancers; thus, our remaining IHC assessments were performed with CD163 data.

Previous clinical studies have shown a correlation between TAM density and cancer prognosis. Most studies have shown a significant correlation between TAM density and poor prognosis, especially among breast, thyroid, and lung cancers. , $7,8,22^{2}$ 
However, several studies have reported that TAM density is associated with good prognosis. Such contradictory results might be due to differences in the number, grade, stage, or size of tumors among the studies. In addition, previous clinical studies have used various methods to assess TAM infiltration. The use of different approaches could have also contributed to the inconsistent results. Although the sample size for each cancer subtype in our study population was small, the results in this study comparing TAM density in various human cancers are significant; therefore, further studies with larger sample sizes are warranted.

In summary, we detected a trend toward an inverse correlation between CD163-positive macrophage density and five-year survival rate in 14 different types of human cancers. In particular, PTC and ATC clearly showed an inverse correlation between TAM density and prognosis. Our results suggest that TAM density is a potential biomarker of poor prognosis in human cancers.

\section{Conflicts of Interest}

No potential conflict of interest relevant to this article was reported.

\section{Acknowledgments}

This work was supported by a grant from the Next-Generation BioGreen 21 Program (No.PJ00954003), Rural Development Administration, Republic of Korea.

\section{REFERENCES}

1. Coussens LM, Werb Z. Inflammation and cancer. Nature 2002; 420: 860-7.

2. Cho SW. Interactions between immune cells and tumor cells. J Korean Thyroid Assoc 2013; 6: 96-100.

3. Zhang QW, Liu L, Gong CY, et al. Prognostic significance of tumorassociated macrophages in solid tumor: a meta-analysis of the literature. PLoS One 2012; 7: e50946.

4. Bingle L, Brown NJ, Lewis CE. The role of tumour-associated macrophages in tumour progression: implications for new anticancer therapies. J Pathol 2002; 196: 254-65.

5. Kim S, Cho SW, Min HS, et al. The expression of tumor-associated macrophages in papillary thyroid carcinoma. Endocrinol Metab (Seoul) 2013; 28: 192-8.

6. Zeni E, Mazzetti L, Miotto D, et al. Macrophage expression of interleukin-10 is a prognostic factor in nonsmall cell lung cancer. Eur Respir J 2007; 30: 627-32.

7. Campbell MJ, Tonlaar NY, Garwood ER, et al. Proliferating macrophages associated with high grade, hormone receptor negative breast cancer and poor clinical outcome. Breast Cancer Res Treat 2011; 128: 703-11.

8. Sato S, Hanibuchi M, Kuramoto T, et al. Macrophage stimulating protein promotes liver metastases of small cell lung cancer cells by affecting the organ microenvironment. Clin Exp Metastasis 2013; 30: $333-44$

9. Forssell J, Oberg A, Henriksson ML, Stenling R, Jung A, Palmqvist $R$. High macrophage infiltration along the tumor front correlates with improved survival in colon cancer. Clin Cancer Res 2007; 13: 1472-9.

10. Ohno S, Inagawa $\mathrm{H}$, Dhar DK, et al. The degree of macrophage infiltration into the cancer cell nest is a significant predictor of survival in gastric cancer patients. Anticancer Res 2003; 23: 5015-22.

11. Welsh TJ, Green RH, Richardson D, Waller DA, O'Byrne KJ, Bradding P. Macrophage and mast-cell invasion of tumor cell islets confers a marked survival advantage in non-small-cell lung cancer. J Clin Oncol 2005; 23: 8959-67.

12. Kawai $\mathrm{O}$, Ishii G, Kubota $\mathrm{K}$, et al. Predominant infiltration of macrophages and CD8(+) T cells in cancer nests is a significant predictor of survival in stage IV nonsmall cell lung cancer. Cancer 2008; 113: $1387-95$

13. Ohno S, Ohno Y, Suzuki N, et al. Correlation of histological localization of tumor-associated macrophages with clinicopathological features in endometrial cancer. Anticancer Res 2004; 24: 3335-42.

14. Cho SW, Kim YA, Sun HJ, et al. Therapeutic potential of Dickkopf-1 in wild-type BRAF papillary thyroid cancer via regulation of betacatenin/E-cadherin signaling. J Clin Endocrinol Metab 2014; 99: E1641-9.

15. Jung KW, Won YJ, Kong HJ, Oh CM, Lee DH, Lee JS. Cancer statistics in Korea: incidence, mortality, survival, and prevalence in 2011. Cancer Res Treat 2014; 46: 109-23.

16. Cornett WR, Sharma AK, Day TA, et al. Anaplastic thyroid carcinoma: an overview. Curr Oncol Rep 2007; 9: 152-8.

17. Are C, Shaha AR. Anaplastic thyroid carcinoma: biology, pathogenesis, prognostic factors, and treatment approaches. Ann Surg Oncol 2006; 13: 453-64.

18. Smallridge RC, Copland JA. Anaplastic thyroid carcinoma: pathogenesis and emerging therapies. Clin Oncol (R Coll Radiol) 2010; 22: 486-97.

19. Haymart MR, Banerjee M, Yin H, Worden F, Griggs JJ. Marginal treatment benefit in anaplastic thyroid cancer. Cancer 2013; 119: 3133-9.

20. Lin JD, Chen ST, Hsueh C, Chao TC. A 29-year retrospective review of papillary thyroid cancer in one institution. Thyroid 2007; 17: 535-41.

21. Murri AM, Hilmy M, Bell J, et al. The relationship between the systemic inflammatory response, tumour proliferative activity, T-lym- 
phocytic and macrophage infiltration, microvessel density and survival in patients with primary operable breast cancer. Br J Cancer 2008; 99: 1013-9.

22. Medrek C, Pontén F, Jirström K, Leandersson K. The presence of tumor associated macrophages in tumor stroma as a prognostic marker for breast cancer patients. BMC Cancer 2012; 12: 306.

23. Ohtaki Y, Ishii G, Nagai K, et al. Stromal macrophage expressing CD204 is associated with tumor aggressiveness in lung adenocarcinoma. J Thorac Oncol 2010; 5: 1507-15.

24. Zhang BC, Gao J, Wang J, Rao ZG, Wang BC, Gao JF. Tumor-associated macrophages infiltration is associated with peritumoral lymphangiogenesis and poor prognosis in lung adenocarcinoma. Med Oncol 2011; 28: 1447-52.

25. Ma J, Liu L, Che G, Yu N, Dai F, You Z. The M1 form of tumor-associated macrophages in non-small cell lung cancer is positively associated with survival time. BMC Cancer 2010; 10: 112.
26. Al-Shibli K, Al-Saad S, Donnem T, Persson M, Bremnes RM, Busund LT. The prognostic value of intraepithelial and stromal innate immune system cells in non-small cell lung carcinoma. Histopathology 2009; 55: 301-12.

27. Steele RJ, Eremin O, Brown M, Hawkins RA. Oestrogen receptor concentration and macrophage infiltration in human breast cancer. Eur J Surg Oncol 1986; 12: 273-6.

28. Pollard JW. Tumour-educated macrophages promote tumour progression and metastasis. Nat Rev Cancer 2004; 4: 71-8.

29. Heusinkveld M, van der Burg SH. Identification and manipulation of tumor associated macrophages in human cancers. J Transl Med 2011; 9: 216.

30. Ambarus CA, Krausz S, van Eijk M, et al. Systematic validation of specific phenotypic markers for in vitro polarized human macrophages. J Immunol Methods 2012; 375: 196-206. 

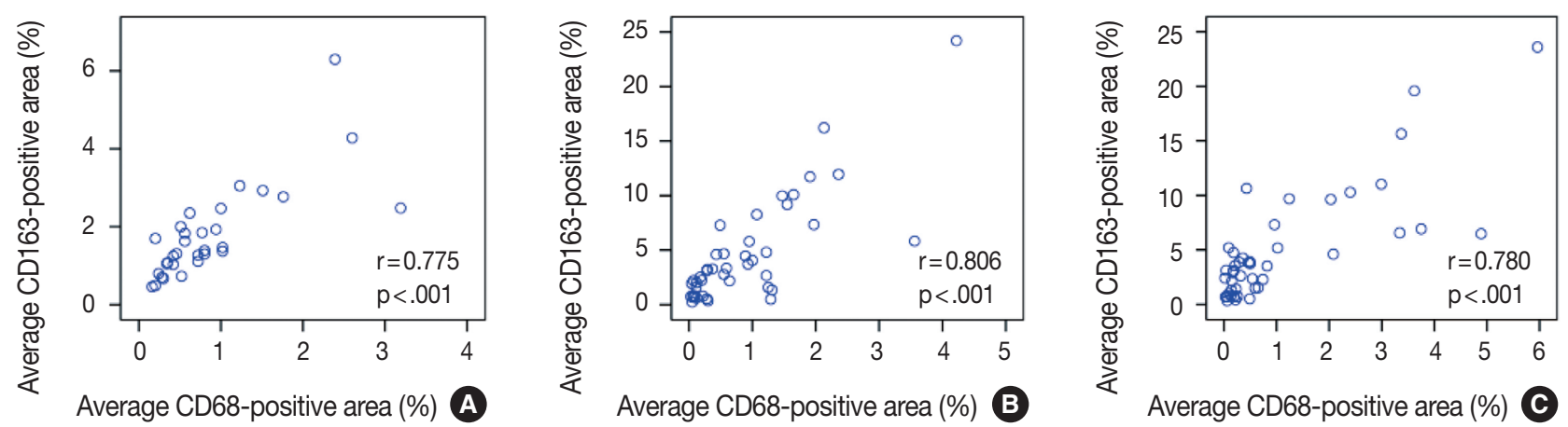

Appendix 1. The correlation between average CD163-positive area (\%) and average CD68-positivie area (\%). (A) Thyroid cancer. (B) Breast cancer. (C) Lung cancer.

Appendix 2. Average CD163-positive area (\%) according to clinicopathologic characteristics in lung, breast, and thyroid cancer

\begin{tabular}{|c|c|c|c|}
\hline Charaacteristic & No. & CD163 (\%) & $p$-value \\
\hline \multicolumn{4}{|l|}{ Lung } \\
\hline \multicolumn{4}{|l|}{ Histology } \\
\hline Adenocarcinoma & 19 & $5.8 \pm 4.4$ & .428 \\
\hline SQCC & 20 & $5.9 \pm 4.5$ & \\
\hline Large cell carcinoma & 4 & $8.7 \pm 7.3$ & \\
\hline SCC & 2 & $1.0 \pm 0.5$ & \\
\hline Mixed carcinoma & 4 & $5.0 \pm 2.3$ & \\
\hline \multicolumn{4}{|l|}{ Stage $^{b}$} \\
\hline I & 16 & $5.7 \pm 4.4$ & .821 \\
\hline$\|$ & 12 & $6.6 \pm 5.2$ & \\
\hline III & 10 & $5.5 \pm 3.8$ & \\
\hline IV & 1 & 2.4 & \\
\hline \multicolumn{4}{|l|}{ Survival } \\
\hline Alive & 15 & $3.6 \pm 3.4$ & .104 \\
\hline Death & 15 & $5.9 \pm 3.9$ & \\
\hline \multicolumn{4}{|l|}{ Breast } \\
\hline Infiltrating ductal & 45 & $4.0 \pm 3.4$ & .055 \\
\hline Medullary & 2 & $9.3 \pm 3.4$ & \\
\hline Sarcomatoid & 1 & 1.9 & \\
\hline Metaplastic & 1 & 10.1 & \\
\hline \multicolumn{4}{|l|}{ Stage $^{b}$} \\
\hline$\| \mathrm{A}$ & 13 & $5.3 \pm 4.9$ & .060 \\
\hline$\| \mathrm{B}$ & 10 & $4.2 \pm 2.4$ & \\
\hline$\| I A$ & 12 & $1.8 \pm 1.4$ & \\
\hline IIIB & 10 & $4.5 \pm 2.3$ & \\
\hline Alive & 30 & $4.2 \pm 3.7$ & .726 \\
\hline Death & 6 & $3.7 \pm 2.5$ & \\
\hline \multicolumn{4}{|l|}{ Thyroid } \\
\hline PTC & 35 & $1.8 \pm 1.3$ & $<.001$ \\
\hline ATC & 18 & $22.9 \pm 17.1$ & \\
\hline \multicolumn{4}{|l|}{ Stage ${ }^{b}$} \\
\hline I & 3 & $1.4 \pm 0.6$ & .943 \\
\hline$\|$ & 3 & $1.7 \pm 1.1$ & \\
\hline III & 21 & $1.8 \pm 1.2$ & \\
\hline IV & 4 & $1.8 \pm 0.8$ & \\
\hline Alive & 15 & $1.8 \pm 1.5$ & .241 \\
\hline Death & 2 & $3.3 \pm 2.8$ & \\
\hline
\end{tabular}

SQCC, squamous cell carcinoma; SCC; small cell carcinoma, PTC; papillary thyroid carcinoma, ATC; anaplastic thyroid carcinoma.

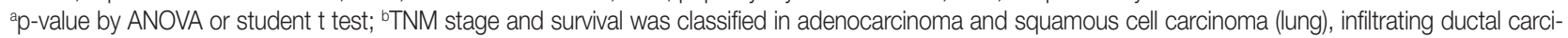
noma (breast) and papillary thyroid carcinoma (thyroid). 